\title{
ARTICLE
}

\section{Medical isotopes from ISAC actinide targets}

\author{
Peter Kunz ${ }^{\mathrm{a}, \mathrm{b} *}$, Corina Andreoiu ${ }^{\mathrm{b}}$, Jason R. Crawford ${ }^{\mathrm{a}, \mathrm{d}}$, Julia Even ${ }^{\mathrm{a}, \mathrm{e}}$, Fatima H. Garcia ${ }^{\mathrm{b}}$, Laura Lambert ${ }^{\mathrm{a}}$, Jens Lassen ${ }^{\mathrm{a}}$, \\ Valery Radchenko ${ }^{\mathrm{a}}$, Caterina F. Ramogida ${ }^{\mathrm{a}}$, Andrew K.H. Robertson ${ }^{\mathrm{a}, \mathrm{c}}$, Thomas J. Ruth ${ }^{\mathrm{a}, \mathrm{d}}$ and Paul Schaffer, \\ ${ }^{a}$ TRIUMF, 4004 Wesbrook Mall, Vancouver, BC V6T 2A3, Canada; ${ }^{b}$ Department of Chemistry, Simon Fraser University, Burnaby, \\ BC V5A 1S6, Canada; ${ }^{c}$ Physics and Astronomy, University of British Columbia, Vancouver, BC, V6T 1Z4, Canada; ${ }^{d}$ Department \\ of Physics and Astronomy, University of Victoria, Victoria, BC, V8P 5C2, Canada: ${ }^{e} \mathrm{KVI}-$ Center for Advanced Radiation \\ Technology, Zernikelaan 25, 9747 AA Groningen, Netherlands
}

\begin{abstract}
The ISAC facility (Isotope Separation and ACceleration) at TRIUMF provides a wide range of radioactive isotope beams (RIB) for research in the fields of nuclear astrophysics, nuclear structure and material science by irradiating ISOL-type (Isotope Separation On-Line) targets with a $480 \mathrm{MeV}$ proton beam from the TRIUMF $\mathrm{H}^{-}$cyclotron. We have developed a compact collection device for the implantation of mass-separated RIB on a target disc at energies between $20-55 \mathrm{keV}$. We also have developed a method to retrieve $>95 \%$ of activity from the implantation target by chemical etching and have investigated alpha recoil separation of ${ }^{213} \mathrm{Bi}$ from a ${ }^{225} \mathrm{Ac} /{ }^{225} \mathrm{Ra}$ implantation source.
\end{abstract}

Keywords: medical isotopes; radioactive isotope beams; ISOL; actinium; actinide targets; radiotherapy

\section{Introduction}

The ISAC (Isotope Separation and ACceleration) facility at TRIUMF facility provides a wide range of radioactive isotope beams (RIB) for research in the fields of nuclear astrophysics, nuclear structure and material science by irradiating ISOL (Isotope Separation On-Line) targets with a $480 \mathrm{MeV}$ proton beam from the TRIUMF H- cyclotron [1].

A more recent application for the facility is the production of radioisotopes for pre-clinical nuclear medicine research. Induced fragmentation, spallation and fission reactions generate a wide range of exotic isotopes spanning almost the whole range of the periodic table of elements. Some of those isotopes are of interest for nuclear medicine applications (imaging, therapy) and can be produced in sufficient quantities for pre-clinical research from a variety of target materials [2]. The current status of verified RIB intensities is published in the ISAC Yield Database [3]. This paper is focusing on the collection of the heavy alpha emitting isotope ${ }^{225} \mathrm{Ac}$ and its radioactive precursor ${ }^{225} \mathrm{Ra}$. Also discussed will be the preparation of ${ }^{213} \mathrm{Bi}$ samples from the ${ }^{225} \mathrm{Ac}$ decay chain via recoil transfer.

\section{Experimental}

The ISAC facility has two target stations which are operated alternately. Within the target station a

*Corresponding author. Email: pkunz@triumf.ca production target is irradiated with a high-intensity proton beam of up to $100 \mu \mathrm{A}$. The maximum achievable beam power is $50 \mathrm{~kW}$ for high-power targets [4], however, the permissible beam power on target depends on the target material properties, the design of the employed target and on regulatory limitations.

Even with reduced proton beam currents only refractory target materials can withstand a typical beam power deposition of several $\mathrm{kW}$ and the associated high temperatures. Target materials are arranged in the cylindrical tantalum target container as stacks of thin foils. At ISAC, either stacks of tantalum or niobium metal foils (foil thickness $25 \mu \mathrm{m}$ ) or composite materials are used. The latter consist of thin layers of refractory oxides or carbides bonded to metal or graphite backing foils, respectively. A foil stack of such a composite material has a better overall thermal conductivity and therefore improved power dissipation compared to the pure oxide or carbide [5]. The production rate for a specific isotope is the product of production cross section, proton beam intensity and target thickness. The thickness range of ISAC targets is between 0.05 to 0.5 $\mathrm{mol} / \mathrm{cm}^{2}$, depending on the target material. The actual isotope beam intensity that arrives at the experiment also depends on the release efficiency from the target, the ionization efficiency and the transport efficiency of the ion beam through the ISAC electrostatic beamline network. Beam transport includes the extraction and acceleration of ions from the ion source to energies between 10-55 keV, subsequent mass separation through a low resolution pre-separator, a high-resolution mass 
separator $(\mathrm{m} / \Delta \mathrm{m} \approx 2000)$ and delivery to the ISAC implantation station. For the ionization process a variety of ion source options are available [6]. Ion beams discussed in the context of this paper were ionized with the surface ion source or the TRIUMF Laser Ion Source (TRILIS). The efficiency of the release of the produced isotopes from the target material matrix to the ion source depends on diffusion and effusion time constants of the produced elements and their preferred state (atom or molecule) within the hot target.

For the generation of actinium and radium isotopes composite ceramic uranium carbide targets [7] with typical thicknesses between 0.05 and $0.1 \mathrm{~mol} / \mathrm{cm}^{2}$ have been bombarded with a $10 \mu \mathrm{A}$ proton beam up to the current license limit of $5000 \mu \mathrm{Ah}$ at an operating temperature of approximately $1950^{\circ} \mathrm{C}$.

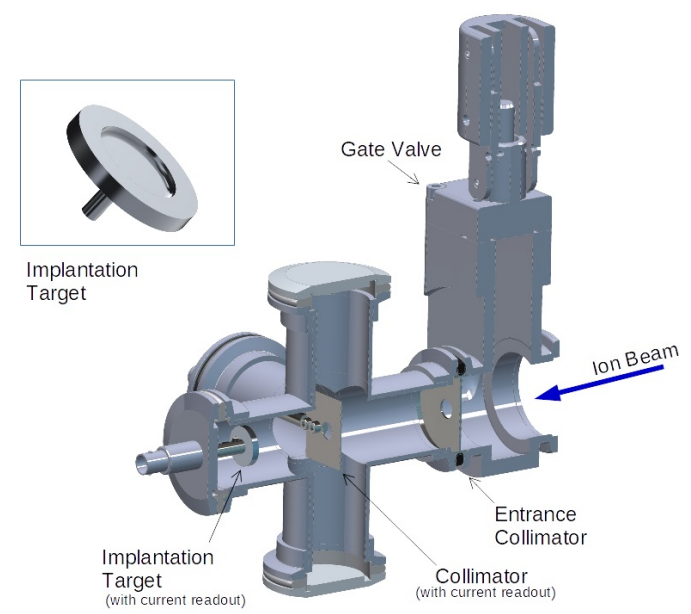

Figure 1. ISAC Implantation Station - Ion beam collection vessel.

After mass separation the RIB is directed to the ISAC Implantation Station (IIS) and implanted on a target in a compact collection device. As depicted in Figure 1, the collection device is a small vacuum vessel based on standard KF40 components. A collimator is used to center the beam on the target which is a modification of a commercial aluminium SEM holder (diameter $25 \mathrm{~mm}$ ). The target is mounted on an electrical feedthrough, enabling RIB current monitoring during the implantation process. The beam current detection limit is in the order of $1 \mathrm{pA}\left(6.24 \cdot 10^{6}\right.$ ions/s) which is well below the intensities of the implantation beams discussed in this paper. A second collimator, to limit the migration of alpha recoils into the beamline, is placed behind the manual gate valve that is used to detach the collection chamber from the beamline. When the implantation is finished, the gate valve at the IIS service port and the manual gate valve at the collection chamber are closed. Then the small volume between the two valves is vented and the collection chamber, still under vacuum, is removed. The whole process takes less than 10 minutes. Up to the point where the chamber is vented within a fume hood or hot cell, the implanted activity can be treated as a closed source.
In the radiochemistry laboratory, the activity, which is implanted at a common ion beam energy of $20 \mathrm{keV}$ about $20 \mathrm{~nm}$ deep in the surface of the aluminium target [8], is removed with a simple etch-rinse cycle. As shown in the insert in Figure 1, a small depression is machined into the implantation target. It is capable of containing about $0.5 \mathrm{ml}$ of liquid.

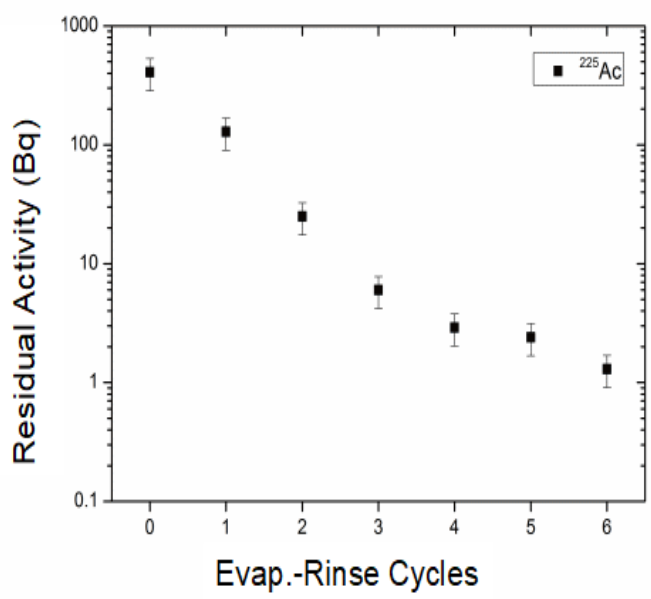

Figure 2. Retrieval of implanted activity with an evaporation rinse cycle.

To remove the activity from the target, $0.1 \mathrm{ml}$ of 0.1 $\mathrm{mol} / \mathrm{l} \mathrm{HCl}$ is filled into the depression and then gently evaporated on a hot plate. After the target has cooled down again, the remaining thin layer of $\mathrm{AlCl}_{3}$ is dissolved with another $0.1 \mathrm{ml} 0.1 \mathrm{~mol} / 1 \mathrm{HCl}$, then the solution is picked up with a micropipette and transferred to a vial. As shown in Figure 2, after consecutive etching and rinse cycles, the residual alpha activity of ${ }^{225}$ Ac was measured with a silicon detector system (Ortec SOLOIST-U0600). Repeating process only 3 times already removes $>95 \%$ of the implanted activity, putting it into a small volume of acidic solution.

Another option of retrieving isotopes from the implantation target is the collection of alpha decay recoils, exploiting the fact that the typical alpha decay recoil energy is with around $100 \mathrm{keV}$ a factor of 2-5 larger than the ion beam implantation energies at ISAC. Therefore, recoils from an alpha decay come out of the surface of the implantation target if their recoil vector points into the right direction. For a thick implantation target, as was used in these experiments, this leads to a theoretical release efficiency of less than $50 \%$ for a simple alpha decay. However, the collection of ${ }^{213} \mathrm{Bi}$ from a ${ }^{225}$ Ac source involves 3 alpha decays in the chain, resulting in a theoretical collection efficiency of $\sim 60 \%$ at an implantation energy of $30 \mathrm{keV}$. This result is based on a recoil track simulation with GEANT4 [9]. The theoretical release efficiencies of ${ }^{213} \mathrm{Bi}$ as a function of implantation energy and the corresponding implantation depths are plotted in Figure 3. In the simulation, all alpha recoils exiting the target are counted as successfully released, including ${ }^{221} \mathrm{Fr}$ and ${ }^{217} \mathrm{At}$, which 
eventually decay into ${ }^{213} \mathrm{Bi}$. Experimentally, a collection efficiency of $35 \%$ was achieved by placing an electrically insulated collection target $3 \mathrm{~mm}$ away from the identical implantation target with the ${ }^{225}$ Ac source. A negative bias of not more than $20 \mathrm{~V}$ on the target opposite from the source is sufficient to collect most of the recoils which, having a recoil energy above the ionization limit, are stopped in air mainly as positive ions. The stopping range of recoils in air under normal pressure is less than $0.1 \mathrm{~mm}$. Aligning the stopping range more accurately with the distance between source and collector by reducing the pressure to $50 \mathrm{mbar}$ and using argon as a buffer gas increased the collection efficiency to $47 \%$.

Based on the yield rates listed in Ref. [3], activities can be extrapolated for a realistic collection period of 2 ISAC beamtime shifts $(24 \mathrm{~h})$. Within this period, a ${ }^{225} \mathrm{Ac}$ beam $\left(\mathrm{T}_{1 / 2}=9.92 \mathrm{~d}\right)$ at a rate of $1.4 \cdot 10^{8} \mathrm{ions} / \mathrm{s}$ from a uranium carbide target / laser ion source combination accumulates an activity of up to $8.6 \cdot 10^{6} \mathrm{~Bq}$. During the same implantation process the accompanying ${ }^{225} \mathrm{Ra}\left(\mathrm{T}_{1 / 2}\right.$ $=14.8 \mathrm{~d}$ ) beam is collected as well. At a rate of $1.7 \cdot 10^{8}$ ions/s from a uranium carbide target / surface ion source combination a total of $1.4 \cdot 10^{13}{ }^{225} \mathrm{Ra}$ atoms can be collected in 24 hours. As ${ }^{225} \mathrm{Ra}$ decays via $\beta^{-}$decay into ${ }^{225} \mathrm{Ac}$, the corresponding maximum activity of $3.3 \cdot 10^{6}$ $\mathrm{Bq}{ }^{225} \mathrm{Ac}$ is reached approximately 18 days after the implantation. One option for using the implanted ${ }^{225} \mathrm{Ac} / \mathrm{Ra}$ activity is to etch off the surface layer of the implantation target using the method outlined above. Then the radium can be separated from the actinium by well-established ion exchange methods. This has been demonstrated as part of the development of multi-isotope SPECT imaging of the ${ }^{225}$ Ac decay chain by Robertson et. al. [10]. Another option is to use the implantation target as a recoil transfer ${ }^{213} \mathrm{Bi}\left(\mathrm{T}_{1 / 2}=45.6\right.$ min) generator. Assuming the aforementioned recoil

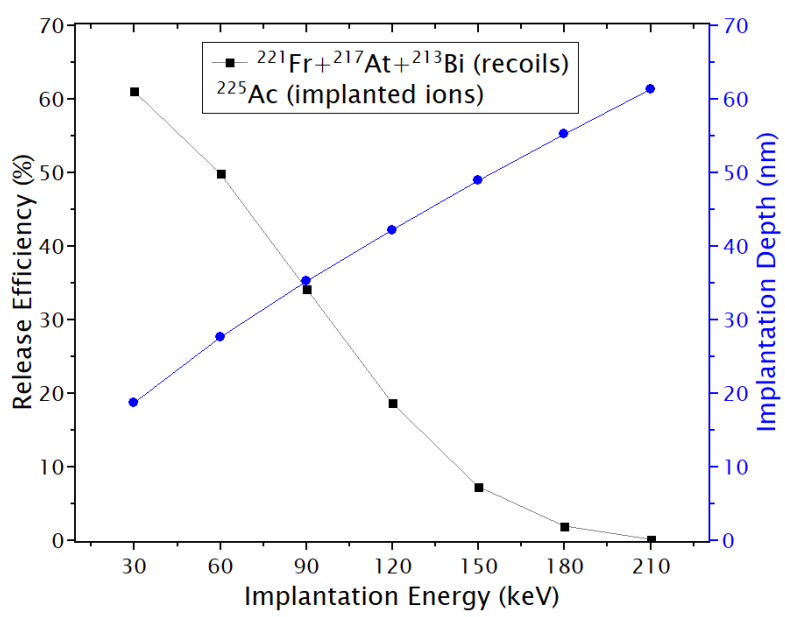

Figure 3. GEANT4 recoil track simulation. The black squares indicate the combined alpha recoil release efficiencies of ${ }^{221} \mathrm{Fr}$, ${ }^{217} \mathrm{At}$ and ${ }^{213} \mathrm{Bi}$ from a ${ }^{225} \mathrm{Ac}$ implantation source as a function of implantation energy (left scale). The blue dots show the associated ${ }^{225} \mathrm{Ac}$ implantation depth based on SRIM [8] (right scale). transfer efficiency of $47 \%$, an activity of $\sim 0.25 \mathrm{MBq}$ ${ }^{213} \mathrm{Bi}$ per $\mathrm{MBq}{ }^{225} \mathrm{Ac}$ can be produced every hour by recoil transfer.

\section{Conclusion}

With the example of ${ }^{225} \mathrm{Ac} / \mathrm{Ra}$ beams, it has been demonstrated that the ISAC facility at TRIUMF has the capability of providing pure isotope samples for pre-clinical nuclear medicine research. Infrastructure for the ISAC Implantation Station and methods for production and retrieval of medical isotopes have been developed. Similar implantation procedures have also been applied to the production of ${ }^{209 / 211}$ At via the implantation of ${ }^{211 / 213} \mathrm{Fr}$ beams for At-based $\alpha$-therapy research $[11,12]$. In principle, the methods described above are applicable for any isotope beam from ISAC targets.

In this paper, only established yield rates (Ref. [3]) and an implantation period limited to 24 hours were used to extrapolate accumulated activities. However, for relatively long-lived isotopes, such as ${ }^{225} \mathrm{Ra}$ and ${ }^{225} \mathrm{Ac}$ larger activities could be obtained by simply increasing the implantation period. Furthermore, beam intensities could be increased by irradiating thicker targets at higher proton beam currents. In particular in the case of actinide targets, theoretical as well as technical limits have not been reached yet. Another option to increase the production of heavy radioisotopes like ${ }^{223-225} \mathrm{Ra}$ and ${ }^{225} \mathrm{Ac}$ is the replacement of uranium with thorium as target material since the production cross sections for these isotopes are significantly higher [13] for thorium. Whether these improvement measures will be pursued and investigated in more detail depends on the demand for higher activities.

\section{Acknowledgements}

TRIUMF receives federal funding via a contribution agreement through the National Research Council of Canada. P. Kunz also acknowledges additional support through a Discovery Grant from the Natural Sciences and Engineering Research Council of Canada (NSERC) SAPIN/00021/2014. Thanks to the ISAC operations crew for their support.

\section{References}

[1] J. Dilling, ISAC and ARIE: The TRIUMF radioactive beam facilities and the scientific program. New York: Springer, 2014.

[2] M. Dombsky and P. Kunz, ISAC targets, Hyperfine Interactions 225, (1-3)(2013), pp. 17-23,

[3] P. Kunz, ISAC Yield Database https://mis.triumf.ca/science/planning/yield/beam. TRIUMF, 2017.

[4] M. Dombsky, P. Bricault and V. Hanemaayer, Increasing beam currents at the TRIUMF-ISAC Facility; techniques and experiences, Nucl. Phys. A, 
746 (2004), pp. 32c-39c.

[5] V. Hanemaayer, P. Bricault and M. Dombsky, Composite ceramic targets for high power proton irradiation, Nuclear Instruments and Methods in Physics Research Section B: Beam Interactions with Materials and Atoms, 266 (19-20) (2008), pp. 4334-4337.

[6] P.G. Bricault et al., Recent developments of the ion sources at Tri University Meson Factory/Isotope Separator and ACcelerator Facility, Review of Scientific Instruments, 83 (2) (2012), p.02A914.

[7] P. Kunz et al., Composite uranium carbide targets at TRIUMF: Development and characterization with SEM, XRD, XRF and L-edge densitometry, Journal of Nuclear Materials, 440 (1-3) (2013), pp. 110-116.

[8] J.F. Ziegler, J.P. Biersack and M.D. Ziegler, SRIM: the stopping and range of ions in matter. Chester, Md: SRIM Co, 2008.

[9] J. Allison et al., Recent developments in Geant 4, Nuclear Instruments and Methods in Physics Research Section A: Accelerators, Spectrometers,
Detectors and Associated Equipment, 835 (2016), pp.186-225.

[10] A.K.H. Robertson et al., Multi-isotope SPECT imaging of the 225 Ac decay chain: feasibility studies, Physics in Medicine and Biology, 62 (11) (2017), pp. 4406-4420.

[11] J.R. Crawford, P. Kunz, H. Yang, P. Schaffer, and T.J. Ruth, $211 \mathrm{Rn} / 211$ At and 209 At production with intense mass separated $\mathrm{Fr}$ ion beams for preclinical 211 At-based $\alpha$-therapy research, Applied Radiation and Isotopes, 122 (2017), pp. 222-228.

[12] J.R. Crawford, H. Yang, P. Kunz, D.S. Wilbur, P. Schaffer, and T.J. Ruth, Development of a preclinical $211 \mathrm{Rn} / 211$ At generator system for targeted alpha therapy research with 211 At, Nuclear Medicine and Biology, 48 (2017), pp. 31-35.

[13] J.W. Engle et al., Cross sections from proton irradiation of thorium at $800 \mathrm{MeV}$, Physical Review C, 88 (1) (2013). 